\title{
THE ADDITIONAL HOSPITAL COSTS GENERATED IN THE MANAGEMENT OF COMPLICATIONS OF PACEMAKER AND DEFIBRILLATOR IMPLANTATIONS
}

\author{
T. Bruce Ferguson, Jr., MD \\ Candice Lilley Ferguson, $\mathrm{MSPH}^{\S}$ \\ Kathryn Crites, RN ${ }^{s}$ \\ Patricia Crimmins-Reda, RN, MSN ${ }^{\S}$
}

The rapid approach of capitated reimbursement mandates that providers examine their practice patterns associated with all surgical procedures. Documentation of (1) the complications associated with these procedures and (2) the additional hospital costs associated with the management of these complications is critical for comprehensive fiscal accountability. This study analyzed (1) the feasibility of obtaining accurate hospital cost data specific for complications and (2) the outcome in terms of fully loaded hospital costs generated in the management of the most common surgical complications associated with pacemaker and nonthoracotomy implantable defibrillator therapies. Between July 1989 and September 1994, a total of 1031 pacemaker and 105 implantable defibrillator procedures were performed by a cardiac surgeon in a tertiary-level teaching hospital setting. The additional fully loaded hospital costs were determined by (1) correlating clinical data from the complete medical record with complete hospital charge data for the admission(s) related to the complication, (2) carving out complication-related charges based on the clinical data, (3) converting complication-related charges to fully loaded costs based on conversion factors in effect at the time of service, and (4) correlating cost with hospital net reimbursement and payor source. The feasibility study determined that accurate and reliable cost data specific to complications can be obtained, although the process was cumbersome and difficult. The outcomes study determined that mean fully loaded complication costs were $\$ 4345 \pm \$ 1540$ for pacemaker lead revision and $\$ 4879 \pm \$ 3167$ for implantable defibrillator lead dislodgment, $\$ 24,459 \pm \$ 14,585$ for pacemaker infection, and $\$ 13,736 \pm \$ 12,505$ for defibrillator generator system malfunction. The one infected defibrillator cost $\$ 57,213$ to treat. Costs exceeded reimbursement for almost all Medicare patients with complications in this study, suggesting that similar shortfalls would occur under a capitation scheme. This information is critical to a complete understanding of the financial impact of interventional procedures in a capitated reimbursement environment. (J THORAC CARDIOVASC SURG 1996;111:742-52)
From the Division of Cardiothoracic Surgery, Department of Surgery, Washington University School of Medicine, and Barnes Hospital at Washington University, St. Louis, Mo.

Read at the Seventy-fifth Annual Meeting of The American Association for Thoracic Surgery, Boston, Mass., April 23-26, 1995.

Received for publication April 27, 1996; revisions requested August 17, 1995; revisions received Dec. 8, 1995; accepted for publication Dec. 13, 1995.

Address for reprints: Dr. T. Bruce Ferguson Jr. MD, Associate Professor of Surgery, Division of Cardiothoracic Surgery, Suite 3108 Queeny Tower, Barnes Hospital Plaza, St. Louis, MO 63110 .

${ }^{\S}$ By invitation.

Copyright 1996 by Mosby-Year Book, Inc.

$0022-5223 / 96 \$ 5.00+0 \quad \mathbf{1 2 / 6 / 7 1 1 6 7}$
0 ver the past 10 years device therapy for cardiac arrhythmias has evolved dramatically, ${ }^{1}$ including the advent of nonthoracotomy implantable cardioverter-defibrillators (NT-ICDs) ${ }^{2-4}$ and dual-chamber, rate-responsive pacemaker systems with modeswitching capabilities. ${ }^{5-7}$ With approximately 350,000 individuals dying of sudden cardiac death each year, the potential impact of ICD therapy in prevention of this lethal event remains extremely high. ${ }^{8}$ The increasing age of the United States population assures that pacing therapy for bradycardia will continue to be a major therapeutic option well into the next century. ${ }^{9}$

Despite their effectiveness,, , 10, 11 interfacing these two high- technology antiarrhythmic therapies into the setting of managed care and capitated 
reimbursement is becoming considerably more difficult. ${ }^{12-14}$ The initial cost of these devices is high, and the life-long nature of the therapy is associated with significant longitudinal cost implications as well.

For reasons related to the broad medical-specialty provider base ${ }^{6}$ and the rapid development of technology, ${ }^{13,14}$ the clinical outcomes after pacemaker and NT-ICD therapy have become somewhat difficult to accurately determine. Even more difficult to determine is the financial impact of acute and chronic complications of these therapies. These costs represent unanticipated and in many instances unrecoverable provider expenditures ${ }^{15}$ in a managed care environment, as suggested by current Medicare reimbursement schemes. ${ }^{16}$

The purpose of this analysis was twofold: first, to determine the feasibility of obtaining accurate and reliable fully loaded cost data specifically related to the management of complications of an interventional procedure; second, to determine the financial outcome (in terms of the additional, fully loaded hospital costs) generated by the appropriate management of complications of pacemaker and ICD therapy at Washington University School of Medicine and Barnes Hospital.

\section{Methods}

Clinical series. The study interval extended between July 1, 1989, and September 1, 1994. During this time interval, 1031 transvenous pacemaker systems were implanted by one of the authors (T.B.F.) at Barnes Hospital, as an integral part of the Washington University Pacemaker Service. ${ }^{7}$ From 1989 to 1991, the majority of implants were performed on an inpatient basis; over the last 3 years of the study interval this shifted to a predominance of outpatient procedures.

During this same time interval, 331 ICD procedures were performed by the same surgeon in conjunction with the members of the Section of Electrophysiology of Barnes Hospital. Of these 331 implants, a total of 105 first-time devices were implanted during the past 2 years as nonthoracotomy systems (NT-ICD), under a variety of clinical investigational protocols sponsored by the Food and Drug Administration.

Identification of complications. All patients were followed up in the Pacemaker Clinic or Defibrillator Clinic at Washington University. Essentially $100 \%$ follow-up was provided for all patients; pacemaker implant and follow-up information were stored online in a customized pacemaker follow-up computer system (Paceart, Inc., Paramus, N.J.), and a similar in-house system was used for the ICD patients. All complications that developed in the follow-up period were identified and managed at Barnes Hospital.
In addition to these in-house patients, a number of patients with preexisting complications were transferred to Barnes Hospital for definitive therapy.

For the purposes of this study, all common complications in the series of patients were analyzed. For pacemaker patients these included (1) lead dislodgment necessitating operative repositioning or lead fracture necessitating replacement for pacemaker implants, (2) infection of the pacemaker system, and (3) generator pocket hematoma formation necessitating operative decompression. For NT-ICD patients these included (1) lead dislodgment or fracture of the nonthoracotomy lead system, (2) system infection, (3) generator malfunction necessitating surgical reexploration/replacement, and (4) system modification owing to threshold elevation necessitating surgical reexploration and revision.

Financial analysis. Both in-house and transfer patients having one of these complications at any time during the study interval were included in the financial analysis.

Feasibility study. From review of the complete medical record, those aspects of the patient's clinical course that were directly attributable to the complication were identified. In addition, the following variable parameters were identified: hospital length of stay (total LOS), the LOS resulting from treatment of the complication (complication LOS), the number of minutes in the operating room required for any complication-related surgical procedure(s), any device(s) implanted, and any intensive care requirement. At the same time, the hospital bill containing all per diem itemized charges, adjustments, and payment data for the hospitalization during which the complication was treated were obtained from the billing office at Barnes Hospital.

Two financial analysis categories of patients were identified: Type 1 patients were admitted specifically for treatment of the complication (e.g., admission for explantation of an infected pacemaker system), and the charges for the entire hospitalization were included in the analysis; in type 2 patients the complication developed during the hospitalization in which the initial implant was performed (e.g., acute lead dislodgment after pacemaker implantation). The hospital days resulting from treatment of that complication were documented from the clinical data, and all the charges incurred during that time interval in the treatment of that complication were identified and carved out from the total hospital charges for that admission.

Once the charges were identified, the Barnes Hospital finance office used Barnes Hospital financial data software to convert the actual cost incurred by the hospital for that provision of care at the time of service. These hospital costs were fully loaded, and the accuracy of these costs was verified by internal review in the finance office.

Outcome financial analysis for complications of pacemaker and ICD therapy. Total hospital charges, total hospital costs (costs for entire hospitalization), total complication costs, and carve-out complication costs (costs for complication treatment) were determined. Complication costs were defined as beginning when a definitive complication diagnosis was established and ending when inpatient therapy for that complication was satisfactorily completed. In addition, contractual adjustments to revenue, total revenue, and source of revenue (Medicare vs com- 
mercial pay) were documented for each patient. The number of patients in each category of complications in the pacemaker and NT-ICD groups, as well as the mean \pm standard deviation of each financial category, was calculated. Profit/loss was calculated by subtracting total costs from total revenue for type 1 patients only (entire hospitalization was for the complication); carve out of revenues was not verifiable and, therefore, was not performed in the analysis. Charge and cost data are expressed as mean \pm standard deviation.

\section{Results}

Clinical series. The overall clinical results associated with the 1031 pacemaker procedures included 825 new implants (65\% dual-chamber devices), 49 lead repositions, 93 generator changes, 17 explants of infected devices, and 47 other miscellaneous procedures. The 105 NT-ICD implants included primarily investigational systems from different manufacturers.

There were no deaths related to the 1136 procedures included in the study (Table I).

The overall in-house incidence of pacemaker system infection was $0.58 \%(6 / 1031)$. An additional 11 patients with pacemakers were transferred from outside institutions for definitive therapy of infected systems. The in-house lead reposition/replacement rate was $4.1 \%$ (37/902 procedures involving lead placement); an additional 12 patients were transferred from outside institutions for definitive therapy of lead-related complications. Pocket hematomas necessitating evacuation developed in four patients with pacemakers; all patients were receiving systemic anticoagulation for other medical conditions, and all were over-anticoagulated in restarting their medication after implantation.

One NT-ICD system infection occurred (1/105, $0.95 \%$ ) at the site of a subcutaneous patch in a patient randomized to a lead-plus-patch protocol. The entire ICD system was explanted and, after appropriate antibiotic and empiric amiodarone therapy, a new, lead-only NT-ICD system was implanted.

Four patients had endocardial lead dislodgments $(n=2)$ or fractures $(n=2)$, requiring reexploration with repositioning or extraction and replacement. One additional patient had radiologically identified lead migration and incisional drainage necessitating antibiotic therapy. ICD system sensing problems necessitating reexploration developed in two patients; generator malfunction necessitated device replacement in one of these patients.

Two additional patients, one receiving systemic anticoagulation for severely depressed ventricular function and the other receiving dialysis for chronic renal failure, had pocket hematomas that necessitated reexploration. In both cases the routine postimplantation test was performed as part of this procedure, and in both patients an acute rise in the defibrillation threshold was demonstrated. This rise in defibrillation thresholds necessitated lead repositioning in one and the addition of a subcutaneous patch to the system in the second.

Feasibility study. The ability of the finance office of Barnes Hospital to obtain accurate cost data before mid-1991 was limited. Therefore, 14 of the 49 patients having total pacemaker lead repositioning who were treated before this time were excluded from the financial analysis. A previous study ${ }^{7}$ had determined accurate cost data for patients having a pacemaker infection and being treated before 1991, and these data were included in the analysis. Accurate fully loaded cost data were obtained on the remaining patients with pacemaker and ICD complications.

The process of retrospective data acquisition, correlation of clinical and charge data, determination of carve-out charges, and conversion to costs was a tedious and difficult one, with many determinations and conversions made on an item-by-item basis.

Outcome study. Approximately $70 \%$ of the overall pacemaker implant population of 1031 patients were Medicare recipients; this percentage was slightly higher in the complication group included in the financial analysis. The mean total LOS and complication LOS was longest for the infection subgroup; the added complication LOS for the hematoma subgroup reflected, in part, additional hospital days to manage the patient's anticoagulation status (see Table I).

In the ICD complication group, the number of Medicare recipients was lower, at $44.5 \%$. Complication LOS was longest for the one patient with infection and second longest for the patients with elevated defibrillation thresholds.

Fully loaded hospital costs generated by the treatment of the pacemaker complications are shown in Table II. Note that hospital charges did not accurately reflect any category of true hospital costs; moreover reimbursement, as mean revenue/loss, varied for each complication and between reimbursement sources irrespective of charges or costs. System infection was the most expensive complication, averaging $\$ 24,459 \pm \$ 14,585$ in added costs; these costs were primarily related to the additional 
Table I. Complication data

\begin{tabular}{|c|c|c|}
\hline \multicolumn{3}{|l|}{ Pacemakers } \\
\hline \multicolumn{3}{|l|}{ Procedures $(n=1031)$} \\
\hline \multicolumn{3}{|l|}{$0 \%$ Mortality } \\
\hline In-house pacemaker infections $(n=6)$ & $0.58 \%$ & \\
\hline \multicolumn{3}{|l|}{ Outside referrals $(n=11)$} \\
\hline In-house pacemaker lead related $(n=37)$ & $4.1 \%$ & \\
\hline \multicolumn{3}{|l|}{ Outside referrals $(n=12)$} \\
\hline In-house hematoma $(n=4)$ & $0.38 \%$ & \\
\hline \multicolumn{3}{|l|}{ Complications } \\
\hline \multicolumn{3}{|l|}{ Lead related $(n=49)$} \\
\hline Mean age (yr) & $64.9 \pm 15.4$ & \\
\hline Mean total LOS (days) & $12.5 \pm 7.8$ & Range $1-56$ \\
\hline Mean complication LOS (days) & $2.5 \pm 0.7$ & Range 1-10 \\
\hline \multicolumn{3}{|l|}{ Infection $(n=17)$} \\
\hline Mean age (yr) & $63.9 \pm 17.4$ & \\
\hline Mean total LOS (days) & $25.6 \pm 33.9$ & Range 4-134 \\
\hline Mean complication LOS (days) & $15.9 \pm 7.6$ & Range 4-31 \\
\hline \multicolumn{3}{|l|}{ Hematoma $(n=4)$} \\
\hline Mean age (yr) & $63.5 \pm 16.5$ & \\
\hline Mean total LOS (days) & $39.0 \pm 52.7$ & Range 9-118 \\
\hline Mean complication LOS (days) & $5.5 \pm 2.8$ & Range 2-9 \\
\hline \multicolumn{3}{|l|}{ NT-ICD } \\
\hline \multicolumn{3}{|l|}{ Procedures $(n=105)$} \\
\hline \multicolumn{3}{|l|}{$0 \%$ Mortality } \\
\hline Lead related $(n=5)$ & $4.9 \%$ & \\
\hline System malfunction $(n=2)$ & $1.9 \%$ & \\
\hline High DFTs $(n=2)$ & $1.9 \%$ & \\
\hline Infection $(n=1)$ & $0.95 \%$ & \\
\hline \multicolumn{3}{|l|}{ Complications } \\
\hline \multicolumn{3}{|l|}{ Lead related $(n=5)$} \\
\hline Mean age & $44.3 \pm 17.5$ & \\
\hline Mean total LOS (days) & $3.4 \pm 1.6$ & Range 2-6 \\
\hline Mean complication LOS (days) & $2.8 \pm 0.8$ & Range 2-4 \\
\hline \multicolumn{3}{|l|}{ System malfunction $(n=2)$} \\
\hline Mean age & $69.5 \pm 10.6$ & \\
\hline Mean total LOS (days) & $9.0 \pm 7.1$ & Range 4-14 \\
\hline Mean complication LOS (days) & $2.5 \pm 2.1$ & Range 1-4 \\
\hline \multicolumn{3}{|l|}{ High DFTs $(n=2)$} \\
\hline Mean age & $46.5 \pm 24.7$ & \\
\hline Mean total LOS (days) & $21.5 \pm 3.5$ & Range 19-24 \\
\hline Mean complication LOS (days) & $11.0 \pm 4.2$ & Range 8-14 \\
\hline \multicolumn{3}{|l|}{ Infection $(n=1)$} \\
\hline Age & 52 & \\
\hline Mean total LOS (days) & 19 & \\
\hline Mean complication LOS (days) & 19 & \\
\hline
\end{tabular}

LOS, Length of hospital stay; total LOS, LOS for entire hospitalization; complication LOS, hospital days beginning at the time a definitive complication diagnosis was established and ending with completion of therapy for that complication; DFTs, defibrillation thresholds.

LOS required for antibiotic therapy, the replacement pacemaker systems required, and the use of cardiopulmonary bypass for removal of intracardiac infected material $(n=4$ patients). Carve-out costs in the two patients with additional medical problems and prolonged hospitalizations were higher, at $\$ 34,987 \pm \$ 7,795$. The type 1 costs were $\$ 23,056 \pm$ $\$ 14,859$.

Hematoma evacuation and lead-related complications were less expensive. Carve-out costs for the four patients in whom a hematoma developed were $\$ 6187 \pm \$ 2631$; because of the small number of patients, type 1 costs were not determined. The added carve-out cost for the 35 lead-related complications was $\$ 4345 \pm \$ 1540$.

In Fig. 1 the profit/loss data for type 1 patients undergoing pacemaker lead reposition are compared with the reimbursement amount by payor, calculated as reimbursement minus type 1 cost. The analysis for the total lead-related group is shown as 
Table II. Cost of pacemaker complications

\begin{tabular}{|c|c|}
\hline \multicolumn{2}{|l|}{$\begin{array}{l}\text { Complications with complete } \\
\text { financial data }(n=56)\end{array}$} \\
\hline Total aggregate charges & $\$ 3,131,341$ \\
\hline Total hospital costs & $\$ 1,708,890$ \\
\hline Mean & $\$ 30,516 \pm \$ 38,755$ \\
\hline Total complication costs & $\$ 592,664$ \\
\hline Mean & $\$ 10,583 \pm \$ 12,222$ \\
\hline \multicolumn{2}{|l|}{ Lead related $(n=35)$} \\
\hline Total charges & $\$ 1,590,270$ \\
\hline Mean & $\$ 45,436 \pm \$ 53,757$ \\
\hline Total hospital costs & $\$ 872,579$ \\
\hline Mean & $\$ 24,931 \pm \$ 29,535$ \\
\hline Total complication costs & $\$ 152,106$ \\
\hline Mean & $\$ 4,345 \pm \$ 1,540$ \\
\hline Type 2 costs $(n=21)$ & $\$ 90,376$ \\
\hline Mean & $\$ 4,303 \pm \$ 1,442$ \\
\hline Type 1 costs $(n=14)$ & $\$ 61,730$ \\
\hline Mean & $\$ 4,409 \pm \$ 1,732$ \\
\hline Mean revenue/loss & $\$ 1,706 \pm \$ 2,272$ \\
\hline Medicare $(n=8)$ & $\$ 1,650 \pm \$ 2,983$ \\
\hline C Pay $(n=6)$ & $\$ 1,780 \pm \$ 974$ \\
\hline \multicolumn{2}{|l|}{ Infection $(n=17)$} \\
\hline Total charges & $\$ 1,282,017$ \\
\hline Mean & $\$ 75,413 \pm \$ 100,663$ \\
\hline Total hospital costs & $\$ 690,172$ \\
\hline Mean & $\$ 40,598 \pm \$ 52,189$ \\
\hline Total complication costs & $\$ 415,808$ \\
\hline Mean & $\$ 24,459 \pm \$ 14,585$ \\
\hline Type 2 costs $(n=2)$ & $\$ 69,975$ \\
\hline Mean & $\$ 34,987 \pm \$ 7,795$ \\
\hline Type 1 costs $(n=15)$ & $\$ 345,833$ \\
\hline Mean & $\$ 23,056 \pm \$ 14,859$ \\
\hline Mean revenue/loss & $\$ 5,863 \pm \$ 5,263$ \\
\hline Medicare $(n=10)$ & $\$ 6,354 \pm \$ 5,522$ \\
\hline C Pay $(n=5)$ & $\$ 6,827 \pm \$ 12,477$ \\
\hline \multicolumn{2}{|l|}{ Hematoma $(n=4)$} \\
\hline Total charges & $\$ 64,753$ \\
\hline Mean & $\$ 64,753 \pm \$ 79,270$ \\
\hline Total hospital costs & $\$ 146,139$ \\
\hline Mean & $\$ 36,534 \pm \$ 46,202$ \\
\hline Total complication costs & $\$ 24,750$ \\
\hline Mean & $\$ 6,187 \pm \$ 2,631$ \\
\hline
\end{tabular}

Type I costs, Total complication costs = entire cost of hospitalization; type 2 costs, carve-out complication costs; Medicare, Medicare reimbursement for type 1 patients only; C Pay, commercial payor reimbursement for type 1 patients only.

well. The hospital lost money as a whole on this population of patients; a small profit was made for all patients for both Medicare and commercial pay reimbursement sources, but the standard deviation of these data was large.

Fig. 2 illustrates the profit/loss data for patients treated for pacemaker infections. Again, the total cost exceeded reimbursement in this group of 17 patients. In the 15 type 1 patients, the average loss was $\$ 5863 \pm \$ 5263$ for the hospital; this loss was exaggerated in the Medicare subgroup. Commercial payor reimbursement resulted in a mean profit, but the majority of these patients were treated in the early years of the study when cost shifting was more favorable than at present.

The fully loaded costs incurred in the treatment of NT-ICD complications were considerably higher (Table III). The one patient with an infected system required two hospitalizations and reimplantation of an entirely new endocardial system; this complication resulted in actual costs of $\$ 57,213$. Both patients with hematomas and elevated defibrillation thresholds required additional operating room procedures and had prolonged hospitalizations. Their treatment resulted in a mean additional cost of $\$ 38,864 \pm \$ 4,069$. Reexploration for a generator system malfunction was expensive- $\$ 13,736 \pm$ $\$ 12,505$; this was in part due to the necessity of replacing the ICD device in one of these two patients. The costs incurred for lead repositioning or replacement were similar to those for pacemaker lead problems- $\$ 4879 \pm \$ 3167$. Interestingly, the frequency of this complication with both therapies was similar as well.

Fig. 3 illustrates the cost/reimbursement analysis for the NT-ICD complications in aggregate, because of the small number of patients with each complication. Treatment of both Medicare $(n=3)$ and commercial pay $(n=4)$ type 1 patients resulted in a loss for the hospital $(\$ 1,164 \pm \$ 15,678$ and $\$ 847 \pm$ $\$ 5496$, respectively).

\section{Discussion}

The purpose of this study was twofold: (1) to determine the feasibility of acquiring accurate fully loaded cost data pertaining to treatment of complications of interventional procedures and (2) to apply this approach in a financial outcomes study determining the accurate, fully loaded hospital costs associated with the management of the most common complications of pacemaker and NT-ICD therapy.

Feasiblity study. Determination of accurate fully loaded costs for hospitalizations has been difficult. ${ }^{17}$ Hospital accounting practices have historically been based on charges. In certain academic centers where the medical school did not own the teaching hospital, much of this charge information was considered proprietary. Cost shifting to cover losses incurred in treating uninsured patients made hospital charges even less reflective of true incurred costs. Medicare and third-party payors placed restrictions on the min- 


\section{Profit / Loss}

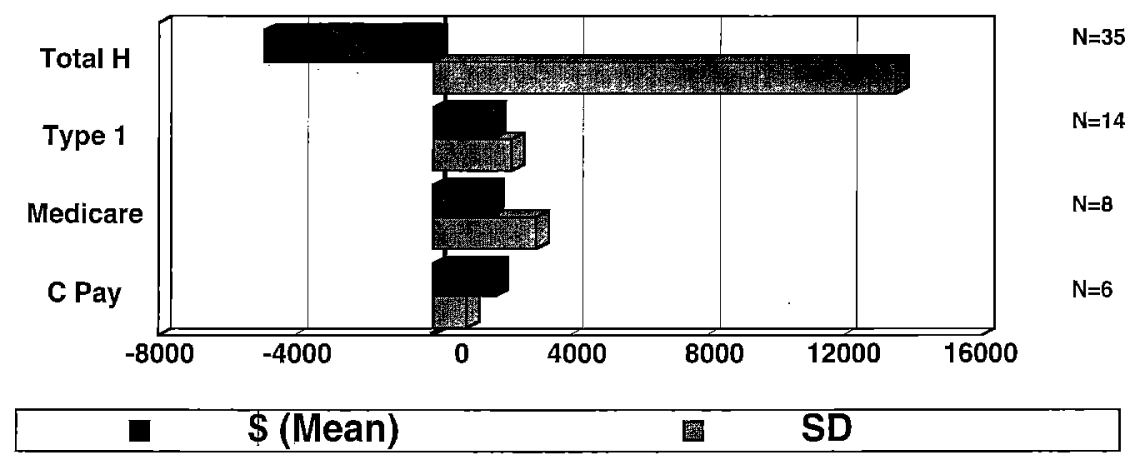

Fig. 1. Profit/loss analysis for pacemaker lead repositions, calculated as net revenue minus complication cost for the categories on the ordinate. Total $H$, total hospital costs (including both type 1 and type 2 patients and including complication and non-complication-related costs; similarly, reimbursement is for the entire hospitalization irrespective of payor); Type 1, type 1 costs (where entire hospitalization was for treatment of the complication); Medicare, those type 1 patients whose costs were reimbursed by Medicare; $C$ Pay, those type 1 patients whose costs were reimbursed by commercial payors; $N$, number of patients in each category; $S D$, standard deviation, expressed as a positive value only for emphasis.

\section{Profit / Loss}

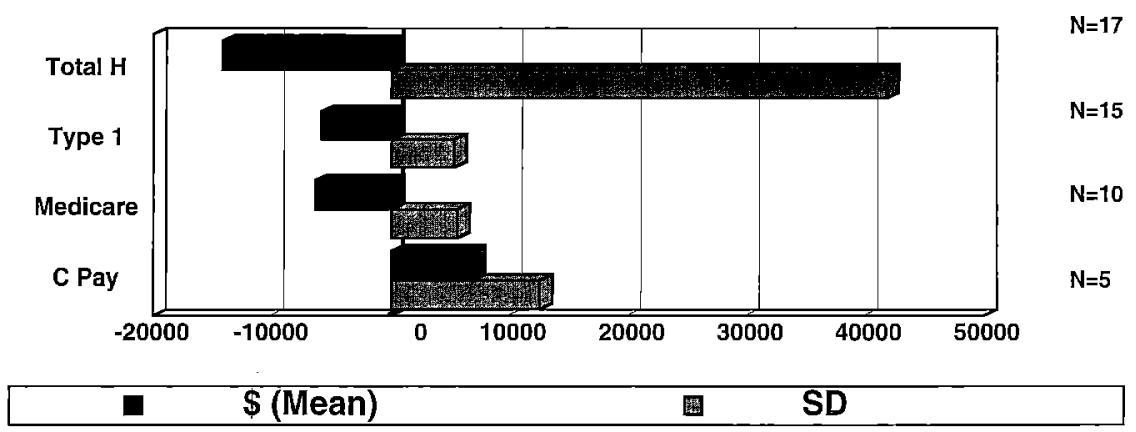

Fig. 2. Profit/loss analysis for pacemaker infection, calculated as net revenue minus complication cost for the categories on the ordinate. Data are expressed as mean complication costs \pm standard deviation (SD); standard deviations are expressed as positive values only for emphasis. For abbreviations see Fig. 1.

imum as well as maximum that hospitals can charge for certain services, further complicating determination of true costs from these charge data. ${ }^{16,18}$ Overall, as demonstrated in this and other studies, charge data are of little value in assessing the true financial impact of a procedure. ${ }^{19}$

Even more difficult has been determination of the fully loaded costs associated with specific complications of therapies or procedures. This is particularly problematic when the complications arise during an admission for an unrelated medical problem (e.g., carve-out complication costs).
The present study demonstrates that accurate cost data can be obtained for complications of pacemaker and NT-ICD therapy. Furthermore, these complication-generated cost data can be carved out of otherwise medically complex hospitalizations. The reliability of this information is dependent on the accurate combination of clinical data from the medical record with relevant financial information. This same approach could be used, for example, to determine the costs associated with reexploration for bleeding after cardiac operations or complications related to interventional cardiovascular procedures. 


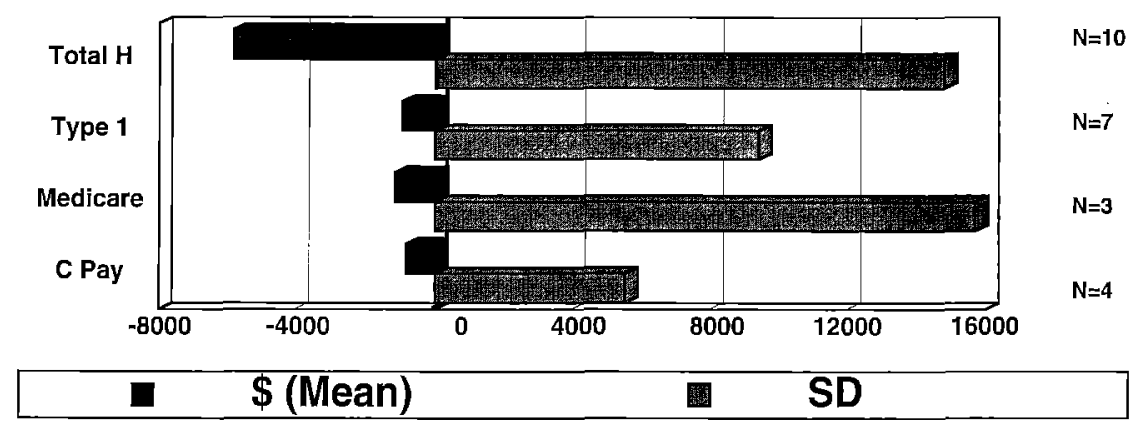

Fig. 3. Profit/loss analysis for all NT-ICD complications, calculated as net revenue minus complication cost for the categories on the ordinate. Data are expressed as mean complication costs \pm standard deviation (SD); standard deviations are expressed as positive values only for emphasis. For abbreviations see Fig. 1.

The study also suggests the need for simplified, prospective methods for determining cost estimates that are accurate and reliable and that can be used for prospective fiscal planning. ${ }^{20-22}$ For at least two reasons, recent changes in the nation's health care system have abruptly made acquisition of these financial outcomes data critical. First, providers are now becoming legally, medically, and financially accountable for cost, quality, and outcomes. ${ }^{23}$ Managed care contracting has altered the financial liability of providers; for example; so-called case-cost contracting has led to the offering of guarantees for certain surgical procedures, covering all complications for a specified period of time after the initial procedure. ${ }^{24}$ Capitation-based programs are offering risk-bearing contracts, taking into account the frequency of the need for an intervention in a managed care population, as well as case and complication costs. For either contractual arrangement to be financially feasible for the provider, accurate determination of the incidence and the costs associated with appropriate treatment of the complications is mandatory.

Second, the decision to purchase services from providers under managed care contractural conditions is most frequently made by assessment of initial costs for procedures and diseases, ${ }^{19}$ with much less emphasis placed on the longitudinal outcomes of those procedures. In this scheme, however, complications represent unanticipated future expenses representing potential financial shortfalls for the provider. Thus quality of service, defined in this case as minimalization of both acute and chronic procedure-related complications, assumes a new financial implication for providers of health care.
Outcomes study. The present study has examined in a financial outcomes analysis the most common complications associated with pacemaker and NTICD therapy. Reasons for choosing these procedures include the expertise available in these therapies at Washington University, large implant patient populations for these devices, uniformity of care with a single surgeon implanting all devices, and complete follow-up. ${ }^{1,7}$

The study has documented that treatment of these complications is expensive. For the duration of the study, 1989 to 1994 , the total fully loaded cost incurred by Barnes Hospital for treatment of all of these complications (excluding the 14 patients having pacemaker lead reposition before 1991) was $\$ 779,804$. Moreover, when the total hospital costs were compared with reimbursement, the hospital lost money on both pacemaker (Figs. 1 and 2) and NT-ICD patients (Fig. 3); this reflects the general clinical and payor status of many of these device recipients.

Infection of device systems was the most expensive complication, primarily resulting from an extended LOS, associated antibiotic therapy, and the replacement hardware. Not surprisingly, other complications resulting in prolonged LOS or more intensive services resulted in higher fully loaded costs to the hospital.

Of note, the difference between an infection rate for pacemaker implantation of $0.5 \%$ and $5 \%$ represents a difference of approximately $\$ 250,000$ per year in provider costs. Infectious complication rates for pacemaker implantation reported in the literature range from $0.5 \%$ to almost $20 \% .^{7,30-33}$ At the current reimbursement rate for pacemaker implan- 
tation paid by Medicare, this amount represents the equivalent of 10 or 11 new device implants. Even so-called minor complications, such as lead dislodgments, are associated with significant aggregate expense.

Complications associated with NT-ICD devices are considerably more expensive to manage than pacemaker complications. The true incidence of NT-ICD complications is difficult to determine from the literature, given the rapid development of this technology and the multiple settings for implantation that are evolving. ${ }^{2,20,21,25}$ Zipes and Roberts ${ }^{20}$ have reported an $18.9 \%$ incidence of invasive complications with endocardial implants (from 2807 patients). Nunain and associates ${ }^{25}$ recently reported an incidence of $36.5 \%$ ICD-related problems with tiered-therapy devices; $13.6 \%$ required some type of system revision at a mean of 8.6 months after the initial implant. Daoud and coworkers ${ }^{26}$ have reported an unexpected late increase in defibrillation thresholds (greater than 10 joules), necessitating system revision, in a small number of patients with NT-ICD systems. These data may be compared with a $10 \%$ incidence in the present series. Although other studies have examined the "cost effectiveness" of ICD therapy as compared with other forms of antiarrhythmic therapy ${ }^{13,27}$ or as a first-line therapy, ${ }^{28}$ the present study is the first to document the actual costs associated with treatment of complications resulting from ICD therapy. The fully loaded cost generated in managing the single infected system in this series was considerably more than the published cost data for initial ICD implants. ${ }^{13,22,29}$ This single ICD infection generated costs equivalent to three elective generator replacements; at Washington University, replacements are done as a surgical outpatient procedure, but still result in costs approaching $\$ 20,000$ per replacement. ${ }^{1,29}$ By way of reference for these cost data, the incidence of complications in the large series of patients examined in this study is equal to or better than other results reported in the literature for pacemaker ${ }^{7,30-33}$ and ICD therapy. ${ }^{2,20,21,25}$ From a financial perspective, it is clear that a significant incidence of any of these complications quickly generates significant additional high fully loaded costs for the provider.

The cost/reimbursement data in this study were generated only from patients whose entire hospitalization was related to the complication, so that the reimbursement amount accurately reflected the entire hospitalization, and the results are a cause for
Table III. Cost of NT-ICD complications

\begin{tabular}{cc}
\hline All complications $(n=10)$ & \\
Total aggregate charges & $\$ 476,932$ \\
Total hospital costs & $\$ 242,162$ \\
Mean & $\$ 24,216 \pm \$ 20,320$ \\
Total complication costs & $\$ 186,810$ \\
Mean & $\$ 16,982 \pm \$ 14,822$ \\
Type 2 complication costs $(n=3)$ & $\$ 82,705$ \\
Mean & $\$ 27,570 \pm \$ 19,771$ \\
Type 1 complication costs $(n=7)$ & $\$ 104,190$ \\
Mean & $\$ 13,023 \pm \$ 11,658$ \\
Mean revenue/loss & $\$ 966 \pm \$ 935$ \\
Medicare $(n=3)$ & $\$ 1,164 \pm \$ 15,678$ \\
Commercial pay $(n=4)$ & $\$ 847 \pm \$ 5,496$ \\
Individual complications & \\
Infection $(n=1)$ & $\$ 57,213$ \\
Mean total cost & $\$ 57,213$ \\
Mean complication cost & \\
Lead rleated $(n=5)$ & $\$ 4,879 \pm \$ 3,167$ \\
Mean totál cost & $\$ 4,879 \pm \$ 3,167$ \\
Mean complication cost & \\
High DFTs $(n=2)$ & $\$ 52,596 \pm \$ 815$ \\
Mean total cost & $\$ 38,864 \pm \$ 4,069$ \\
Mean complication cost & $\$ 32,269 \pm \$ 13,703$ \\
Generator malfunction $(n=2)$ & $\$ 13,736 \pm \$ 12,505$ \\
Mean total cost & \\
Mean complication cost &
\end{tabular}

Type 1 costs, Total complication costs = entire cost of hospitalization; type 2 costs, carve-out complication costs; Medicare, Medicare reimbursement for type 1 patients only; C Pay, commercial payor reimbursement for tyep 1 patients only; DFTs, defibrillation thresholds.

concern. These data (Figs. 1 to 3) demonstrate that a shortfall on reimbursement of Medicare patients was experienced in almost all cases. It is anticipated that these reimbursement levels will continue to be reduced by Congress. ${ }^{8,19,33,34}$ Moreover, the Medicare population will continue to increase substantially in numbers well into the next century; this increase in patient number has been demonstrated to account for the majority of the increase in Medicare expenditures projected over this time interval. ${ }^{34}$

This study focused on complication-related costs of pacemaker and NT-ICD therapy, not the cost of initial implantation. VVI pacemaker placement in all patients and amiodarone therapy in lieu of NT-ICD therapy represent the most dramatic costsaving options with respect to the initial implant procedure. With regard to complications, only two mechanisms exist for reduction of these costs: (1) reduce the incidence of the complications and (2) reduce the cost of treatment of the complications. Multiple studies have documented that the incidence of complications for interventional procedures increases significantly when the operator 
and/or institution performs a subthreshold number of procedures per year. ${ }^{31,32}$ Importantly, the present study also documents that there is an expected financial liability, as well as a quality of care liability, associated with this degree of performance infrequen$\mathrm{cy}{ }^{18}$ particularly with respect to pacemaker and NTICD implantation. Furthermore, this financial liability is present irrespective of such issues as facility sites of implantation and/or the medical speciality of the implanter. Currently, a number of options are available to reduce the cost of treatment of complications. These include shifting to outpatient therapy for intravenous antibiotics, home-health nursing services, and implementation of anticoagulation therapy on an outpatient basis to minimize hospital LOS.

This study has several important limitations. It is a preliminary financial feasibility and outcomes analysis in a selected patient population. The number of patients with complications was small, and the financial analysis was limited to only a 5-year study interval. In a number of patients the devices were implanted under clinical investigational protocols. Although these costs were fully accounted for in this study, current clinical practice is different from several of those protocols. Finally, extremely rapid changes in device technology and health care provision occurred during this study interval.

Nevertheless, the analysis demonstrates directly that management of complications of pacemaker and NT-ICD therapies is expensive. It demonstrates indirectly that complication-related costs can be an important component in the overall cost of device therapy. This is particularly true in the Medicare patient population or under circumstances in which device-related complications are excessively prevalent. Opportunities to reduce the incidence of complications, as well as the additional costs of this therapy, will become increasingly important to providers and managed care organizations. Finally, this identification and quantification is a step toward the accurate financial assessment of this aspect of health care provision that directly links outcomes and costs. As more of the health care system in the United States moves to a managed care environment and toward capitated reimbursement, information such as presented here for pacemaker and ICD therapy will become increasingly important for all types of interventional procedures.

We gratefully acknowledge the expertise and assistance of Mr. Ron Trulove, in the finance office of Barnes Hospital, for his work on this project.

\section{REFERENCES}

1. Ferguson TB Jr. The future of arrhythmia surgery. J Card Electrophysiol 1995;7:621-35.

2. Bardy GH, Hofer B, Johnson G, et al. Implantable transvenous cardioverter-defibrillators. Circulation 1993;87:115268.

3. Moss AJ. Influence of the implantable cardioverter defibrillator on survival: retrospective studies and prospective trials. Prog Cardiovasc Dis 1993;36:85-8.

4. Connoly SJ, Yusuf S. Evaluation of the implantable cardioverter defibrillator in survivors of cardiac arrest: the need for randomized trials. Am J Cardiol 1992;69:959-62.

5. Barold SS, Sanders RS. Rate-adaptive cardiac pacing: cost versus technology versus patient benefit. Am Heart J 1993; 126:1828-34.

6. Bernstein AD, Parsonnet V. Survey of cardiac pacing in the United States in 1989. Am J Cardiol 1992;69:331-8.

7. Ferguson TB Jr, Lindsay BD, Boineau JP. Should surgeons still be implanting pacemakers? Ann Thorac Surg 1994;57: 588-97.

8. Zipes DP. Implantable cardioverter-defibrillator: lifesaver or a device looking for a disease? Circulation. 1994;89:2934-6.

9. Kramer AM. Health care for elderly persons-myths and realities. N Engl J Med 1995;332:1027-8.

10. Griffith MJ, Bexton RS, McComb JM. Financial audit of antitachycardia pacing for the control of recurrent supraventricular tachycardia. Br Heart J 1993;69:272-5.

11. De Belder MA, Linker NJ, Jones S, Camm AJ, Ward DE. Cost implication of the British Pacing and Electrophysiology Group's recommendations for pacing. Br Med J 1992;305: 861-5.

12. Krucoff M, Chu F, McCallum D, Perry S. New medical technologies in a cost containment environment: implantable antitachyarrhythmia devices. PACE 1987;10:2-20.

13. Anderson MH. Predicting the cost-efficacy of implantable cardioverter-defibrillators: use of a model to study new and existing indications. In Luceri RM, ed. Sudden cardiac death: strategies for the 1990s. Miami Lakes: Peritus Corporation, 1992, 159-77.

14. Zipes DP. Are implantable cardioverter-defibrillators better than conventional antiarrhythmic drugs for survivors of cardiac arrest? Circulation 1995;91:2115-8.

15. Ott R, Tanner T, Henderson B, eds. Managed care and the cardiac patient. Philadelphia: Hanley \& Belfus, 1995.

16. Shulkin DJ, Escarce JJ, Enarson C, Eisenberg JM. Impact of the Medicare fee schedule on an academic department of medicine. JAMA 1991;266:3000-3.

17. Giezl HR. Managed care: organizational structures and litigation prospects. In: Ott R, Tanner $\mathrm{T}$, Henderson B, eds. Managed care and the cardiac patient. Philadelphia: Hanley \& Belfus, 1995:9-31.

18. Burstin HR, Lipsitz SR, Udvarhelyi S, Brennan TA. The effect of hospital financial characteristics on quality of care. JAMA 1993;270:845-9.

19. Jordan B. Contracting financial and service matrix. In: Ott R, Tanner T, Henderson B, eds. Managed care and the cardiac patient. Philadelphia: Hanley \& Belfus, 1995:31-8.

20. Zipes DP, Roberts D, PCD Investigators. Results of the world-wide study of the implantable pacemaker, cardioverter-defibrillator: a comparison of epicardial and endocardial lead systems. Circulation [In press].

21. Strickenberger SA, Niebauer M, Man KC, et al. Comparison 
of implantation of nonthoracotomy defibrillators in the operating room versus the electrophysiology laboratory. Am J Cardiol 1995;75:255-7.

22. Williamson BD, Man KC, Niebauer M, et al. The economic impact of transvenous defibrillation lead systems. PACE 1994;17:2297-303.

23. Schleuning SK. Managed care: an overview and prescription for change. In: Ott R, Tanner T, Henderson B, eds. Managed care and the cardiac patient. Philadelphia: Hanley \& Belfus, 1995:3-7.

24. Tanner T, Ott R, Moscoso R, Gutfinger D, Fopiano P. Effective management of global fee services: the integration of interventional and cardiac surgical services. In: Ott R, Tanner T, Henderson B, eds. Managed care and the cardiac patient. Philadelphia: Hanley \& Belfus, 1995:105-14.

25. Nunain SO, Roelke $M$, Trouton $T$, et al. Limitations and late complications of third-generation automatic cardioverterdefibrillators. Circulation 1995;91:2204-13.

26. Daoud E, Niebauer M, Horwood L, et al. Rise in chronic defibrillation threshold necessitating defibrillator lead system revision. J Am Coll Cardiol 1995;23:408A.

27. Kupperman M, Luce BR, McGovern B, Podrid PJ, Bigger JT, Ruskin JN. An analysis of the cost effectiveness of the implantable defibrillator. Circulation 1990;81:91-100.

28. Wever EFD, Hauer RNW, van Capele FJL, et al. Randomized study of implantable defibrillator as first-choice therapy versus conventional strategy in postinfarction sudden death survivors. Circulation 1995;91:2195-203.

29. Ferguson TB Jr. Direct surgery versus ICD therapy for ischemic ventricular tachycardia. Ann Thorac Surg [In press].

30. Phibbs B, Marriott HJL. Complications of permanent transvenous pacing. N Engl $J$ Med 1985;312:1428-32.

31. Parsonnet V, Bernstein AD, Lindsay BD. Pacemaker implantation complication rates: an analysis of some contributing factors. J Am Coll Cardiol 1989;13:917-21.

32. Mansfield PF, Hohn DC, Fornage BD, Gregurich MA, Ota DM. Complications and failures of subclavian-vein catheterization. N Engl J Med 1994;331:1735-8.

33. Allen BJ, Brodsky MA. Cost-effective management of congestive heart failure and cardiac arrhythmias in the managed care setting. In: Ott R, Tanner T, Henderson B, eds. Managed care and the cardiac patient. Philadelphia: Hanley \& Belfus, 1995:229-34.

34. Lubitz J, Beebe J, Baker C. Longevity and Medicare expenditures. N Engl J Med 1995;332:999-1003.

35. Ray SG, Griffith MJ, Jamieson S, Bexton RS, Gold RG. Impact of the recommendations of the British Pacing and Electrophysiology Group on the pacemaker prescription and on the immediate costs of pacing in the Northern Region. Br Heart J 1992;68:531-4.

\section{Discussion}

Dr. D. Glenn Pennington (St. Louis, Mo.). This study involved only hospital cost and not physician reimbursement. Is it not true that a complication would be excluded from all of the current procedural technology codes? In other words, is it not true that all of them refer to changes, for example, after the first 3 weeks?

Dr. Ferguson. That is true in most but not all the cases. Some of these patients were treated several years after the initial event, but those problems still have to be considered complications. When we get to single payment capitation, though, that is not going to be an issue, because we, as providers, are going to be left with the responsibility for treating these complications and we will not be able to bill specifically for them.

Dr. Richard J. Shemin (Boston, Mass.). Dr. Ferguson, in reviewing, have you been able to identify any change in treatment protocols, practice guidelines, or critical pathways for the treatment of these patients? Perhaps more outpatient treatment or other practice style changes would reduce the costs and generate at least a break even or a slight profit for the institution.

Dr. Ferguson. We have attempted to treat these complications as expeditiously as possible. The only complications that really have been most difficult to treat are infectious complications because they require prolonged hospitalizations, and almost all necessitate explantation and reimplantation of expensive hardware. All of the lead repositions and evacuations were done as outpatient procedures.

There was one difference that did come up that was time-related. The slight profit seen in the commercial payors in the infectious pacemaker group was derived from the early years, when cost shifting by those commercial providers was still a possibility. Their profit almost certainly was generated on that basis. In the current environment cost shifting is becoming more difficult, and I think even that subgroup of patients would lose money.

Dr. Agustin Arbulu (Detroit, Mich.). I have a question about the infectious complication, because we had a similar incidence of infections in automatic ICDs. We just had our third infection in over 300 cases that we've done, particularly those with a nonthoracotomy approach, which comprise about two thirds of this population. The problem is that we explanted the device immediately and then consulted our infectious disease experts. Even with the explanted paraphernalia they insisted that the patient undergo 4 to 6 weeks of intravenous antibiotic therapy. The problem is that the patient has the sudden death syndrome. How do you handle those cases? What is your protocol of management?

Dr. Ferguson. That is an extremely difficult situation to manage. The experience with other types of hardware would dictate that those patients do require long-term antibiotic therapy. In the ICD patient population, the only option in most cases is to administer amiodarone for the window of time that they cannot have the ICD as a backup. Currently, that can usually be done on an outpatient basis with home antibiotics, but they all will have to have the ICD reimplanted at some point. There will probably be a small but finite incidence of sudden death in that population.

Dr. Arbulu. Am I correct that you sent them home receiving the antiarrhythmic drugs?

Dr. Ferguson. Yes, unless in the judgment of our electrophysiologists the risk of that was just too high, in which case the patient had to stay in the hospital the entire time.

Dr. Anthony L. Moulton (Providence, R.I.). There is an increasing tendency for pacemakers to be implanted by persons without formal surgical training and in areas 
outside the operating room. Was there as a correlation between your infection rate and the implantation physician? Also, was there a greater expediency with which infectious complications were treated depending on the implanting physician?

Dr. Ferguson. Not based on these data. All of these procedures were done by me at Washington University. There is, at least in the literature, a difference in the infectious complication rate reported in the surgical series as compared with the series reported by implantation in other environments and by cardiologists. The difference is small, but it appears to be real. Although that assessment was not the purpose of this paper, what that means in terms of a hospital administrator is a difference between an infection rate of $0.5 \%$ and $4 \%$ or $5 \%$. That could end up being a difference of $\$ 250,000$ in true hospital costs at the end of a year. Maybe analyses such as these and others can be used to reintroduce the concept that the quality of what is done and not just the initial costs is important in the long term.

\section{ON THE MOVE?}

Send us your new address at least six weeks ahead

Don't miss a single issue of the journal! To ensure prompt service when you change your address, please photocopy and complete the form below.

Please send your change of address notification at least six weeks before your move to ensure continued service. We regret we cannot guarantee replacement of issues missed due to late notification.

\section{JOURNAL TITLE:}

Fill in the title of the journal here.

OLD ADDRESS:

Affix the address label from a recent issue of the journal here.

\section{NEW ADDRESS:}

Clearly print your new address here.

Name

Address

City/State/ZIP
COPY AND MAIL THIS FORM TO:

Journal Subscription Services

Mosby-Year Book, Inc.

11830 Westline Industrial Dr.

St. Louis, MO 63146-3318
OR FAX TO:

314-432-1158

N/ Mosby
OR PHONE:

1-800-453-4351

Outside the U.S., call

314-453-4351 